\title{
Oxygen photo-adsorption related quenching of photoluminescence in group-III nitride nanocolumns
}

\author{
P. Lefebvre , S. Albert, J. Ristić , S. Fernández-Garrido , J. Grandal , \\ M.-A. Sánchez-García, E. Calleja
}

\author{
A B S T R A C T
}

GaN and InGaN nanocolumns of various compositions are studied by room-temperature photoluminescence (PL) under different ambient conditions. GaN nanocolumns exhibit a reversible quenching upon exposure to air under constant UV excitation, following a $t^{1 / 2}$ time dependence and resulting in a total reduction of intensity by $85-90 \%$, as compared to PL measured in vacuum, with no spectral change. This effect is not observed when exposing the samples to pure nitrogen. We attribute this effect to photoabsorption and photodesorption of oxygen that modifies the surface potential bending. InGaN nanocolumns, under the same experimental conditions do not show the same quenching features: The high-energy part of the broad PL line is not modified by exposure to air, whereas a lower-energy part, which does quench by $80-90 \%$, can now be distinguished. We discuss the different behaviors in terms of carrier localization and possible composition or strain gradients in the InGaN nanocolumns. 


\section{Introduction}

In the past decade, semiconductor nanocolumns (NCs) - also called "nanorods" - or nanowires (NWs) based on wide band-gap materials such as $\mathrm{ZnO}$ [1], diamond [2] and group-III nitrides have received increasing interest due to their high aspect ratio and compatibility with silicon-based technology, paving the way to a variety of novel applications [3]. In case of nitride NCs, in addition to superior crystal quality, the main reason for this interest is certainly the potential for improving efficiency and reducing costs of light emitting devices in the visible and ultraviolet ranges of the spectrum [4,5]. Indeed, the nearly-perfect crystal quality of NCs is expected to limit the technological steps that are needed, in planar epilayers, for reducing the densities of nonradiative defects [5-8].

One of the most developed ways to produce vertically aligned, self-assembled III-N NCs is the plasma-assisted molecular beam epitaxy (PA-MBE) [4-11]. This technique [12-15] and also vapor phase deposition methods [16-18] have been used to produce InGaN NCs $[13,14,16,18]$ and nanocolumnar InGaN/GaN quantum heterostructures [12,15,17], providing efficient room-temperature (RT) light emission, probably resulting from carrier localization, as already well-known for InGaN alloys [19]. Emission over the entire visible spectrum was achieved by control of the In incorporation.

Now, the high effective surface of dense NC assemblies suggests that surface should play a much more prominent role than in planar structures. As an example of such surface effects, in undoped GaN planar epilayers, Foussekis et al. [20] recently reported that photo-adsorption of oxygen causes significant quenching of the RT PL. This was explained by the upward bending of the near-surface potential, which is increased by the photo-induced adsorption of species in air ambient. This creates a strong electric field that separates electrons and holes, reducing the effective volume for radiative recombination of free excitons, which is the dominant light emission mechanism in high-quality GaN epilayers at RT. The critical effect of photo-absorption on RT PL intensity has been observed in ZnO NWs [1] as well. It has been showed, also, that surface effects can modify excitonic states at low temperatures [21], e.g. via the build-up of surface-excitons [22]. The existence of the latter was also demonstrated in GaN NWs [23], simultaneously with dramatic ageing effects (radical changes in micro-PL spectra, after long exposure to ambient air) resulting from surface alteration. Surface excitons in GaN NWs and NCs (observed at low temperatures, thus in vacuum) were proved to involve donors randomly distributed at variable distance from the surface $[23,24]$. For completeness, let us mention that surface modifications of transport properties in devices embedding disordered nitride or ZnO NWs, when exposed to certain gases, led to new concepts of gas sensors [25]. In this context, it is important to learn more about possible surface-related effects affecting optical properties of nitride-based NCs.

In this paper, we report on the strong quenching of RT excitonic PL induced by exposing dense ensembles of GaN and InGaN NCs to ambient air, whereas exposure to pure nitrogen has no such effect. We emphasize the significant differences between the two types of NCs, and we discuss the microscopic mechanisms at work.

\section{Samples and experimental details}

Our samples were grown by plasma-assisted molecular beam epitaxy on $\mathrm{Si}(111)$ substrates. Effective N-rich conditions allow for the self-organized growth of NCs with typical densities, heights and diameters of a few $10^{10} \mathrm{~cm}^{-2}, 1.0-1.3 \mu \mathrm{m}$ and $30-50 \mathrm{~nm}$, respectively [11]. We present below the PL results on InGaN NCs grown on top of pure GaN NCs, both parts having similar nominal heights of $\sim 0.6-0.7 \mu \mathrm{m}$. The In content was controlled by the growth temperature: for the chosen $\mathrm{N}, \mathrm{Ga}$ and In fluxes, the growth temperatures were $800^{\circ} \mathrm{C}$ for GaN and between 650 and $750{ }^{\circ} \mathrm{C}$ for InGaN. The lower the growth temperature, the higher the indium incorporation (up to $28 \%$ in our samples) [13]. Detailed studies of the morphology and composition of these NCs, of their growth mechanism and of the temperature-dependent PL spectra will be subject of forthcoming publications.

To introduce this work, let us mention that, at $T=8 \mathrm{~K}$, all our GaN NCs (including the ones on which InGaN NCs were grown) show an intense donor-bound exciton line at $3.4705 \mathrm{eV}$, with FWHM of 2.0-2.5 meV, accompanied by free A and B excitons, easily observable. When the temperature is increased to $300 \mathrm{~K}$, upon ionization of the donor-bound excitons, the free excitons dominate the PL 
spectrum. As for InGaN NCs, their PL features are comparable to those of InGaN epilayers: the linewidth increases with In content, due to enhanced composition fluctuation effects and the PL intensity drops with increasing temperature, but the larger the In composition, the smaller the drop, which can be attributed to increased carrier localization. However, the PL peak energy does not clearly follow the usual s-shaped dependence versus temperature, in the present experimental conditions. Preliminary cathodoluminescence results [26] suggest that the average In content of different NCs can be quite different, thus making complicated the interpretation of T-dependent macroscopic PL spectroscopy.

In the following, we focus on the changes of RT PL when the samples pass from vacuum (pressure of $\sim 10^{-4} \mathrm{~Pa}$ ) to air ambient, under uninterrupted laser excitation. The samples were placed in a chamber where the conditions could be rapidly changed between vacuum and air (or pure nitrogen) at atmospheric pressure and excited by He-Cd laser $\left(\lambda=325 \mathrm{~nm}\right.$ ) with typical power density of $5 \mathrm{~W} \mathrm{~cm}^{-2}$. These conditions are comparable to those of Refs. [1,20].

\section{Results}

Fig. 1 shows the effect of exposure to ambient air on pure GaN NCs. In fact, we observed the same effect in all our GaN NCs, including those followed by InGaN NCs: the intensity of the band-edge excitonic PL quenches by $\sim 85-90 \%$ with no change in spectral shape. The PL intensity drop is fast at the beginning ( $80 \%$ of the signal is lost after $\sim 30 \mathrm{~s}$ ), but it slows down progressively with time: a good fit is given by a $t^{-1 / 2}$ decay, with a residual signal of $\sim 10 \%$ of the initial one, as measured after $2 \mathrm{~h}$ (see the inset in Fig. 1). This quenching dynamics suggests some self-limited chemisorption process in which photo-created free carriers are involved in the adsorption of species at the surface. The accumulation of those species with time raises a potential barrier $[1,20]$ thus hindering further access of free carriers to the surface, which therefore limits additional adsorption.

To confirm the photochemical nature of the process, we checked experimentally that exposure to air alone is not sufficient to induce the quenching effect: starting from vacuum conditions and maximum PL signal, we blocked the laser excitation and exposed the NCs to ambient air for one hour. When the laser was directed again onto the sample (still in air), the PL intensity was not at $10 \%$ of the maximum value, as might be expected from the dynamics shown in Fig. 1. Instead, the signal reached $60 \%$ of its maximum within a few seconds and, then only, it started decaying, finally reaching $10 \%$ of the maximum value. This result is in favor of a photo-adsorption mechanism [20].

The observed effect is reversible: when the sample was placed in vacuum again, under laser excitation, the PL intensity recovered its initial value. This observation suggests the same photodesorption

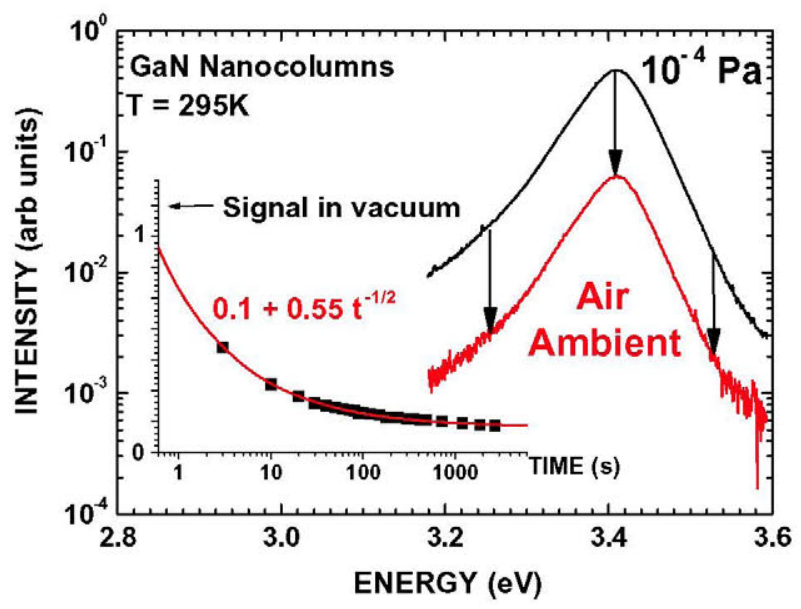

Fig. 1. Quenching of the room-temperature PL from GaN NCs, after exposure to air ambient under uninterrupted He-Cd laser excitation. The spectrum shape is unchanged during the slow decay, which is well fitted by a power law (in $t^{-1 / 2}$ ). 
mechanism as the one proposed in Refs. $[1,20]$. It also agrees with the recent observation of the increase of low-temperature PL intensity of GaN NCs upon continuous UV excitation, also explained [27] by oxygen photodesorption effects.
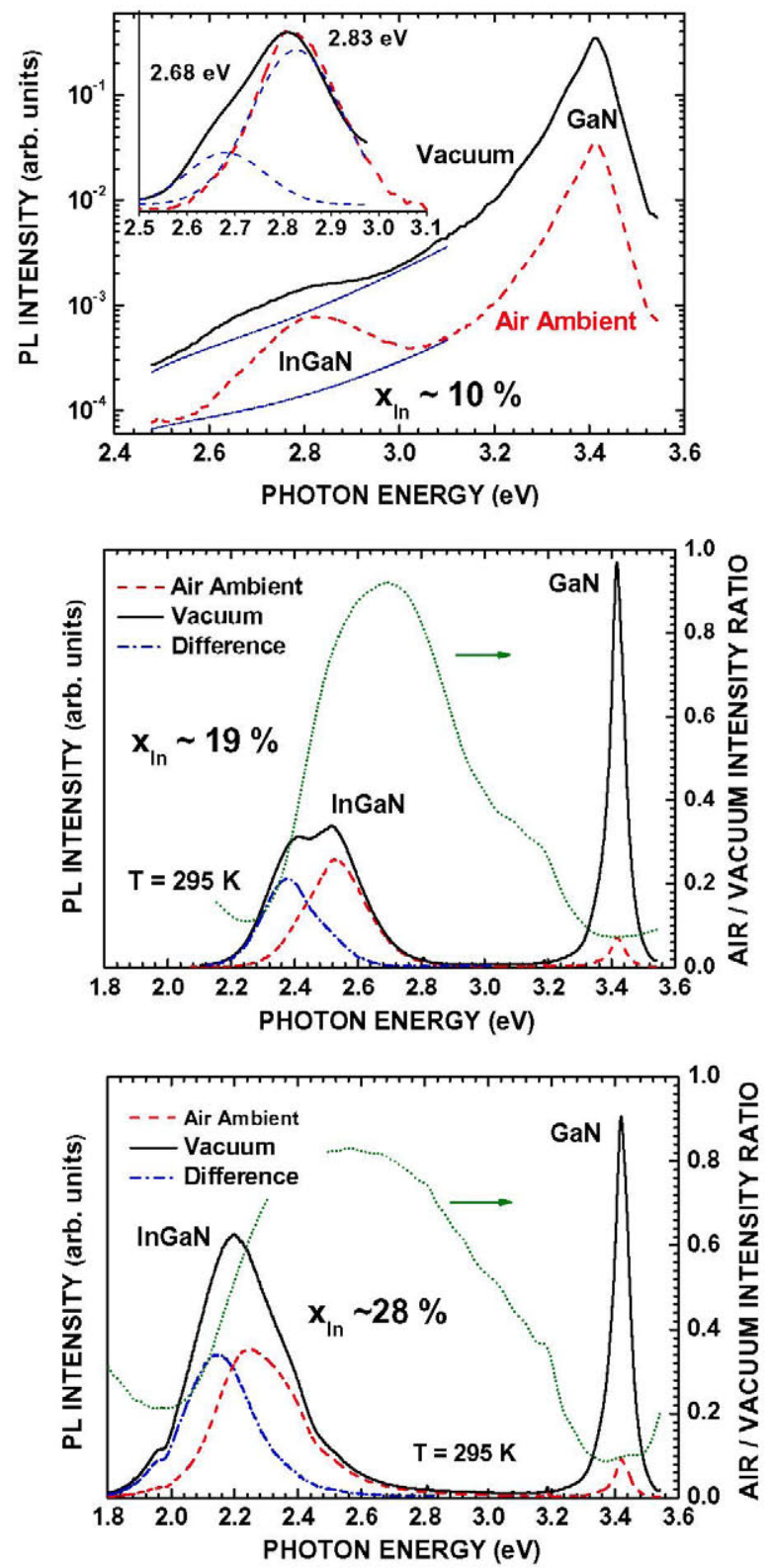

Fig. 2. PL quenching in superposed GaN and InGaN NC samples, with respective InGaN growth temperatures of 725,700 and $675^{\circ} \mathrm{C}$, for (a), (b) and (c), corresponding to the indicated In compositions, estimated from the PL peak position. Black solid curves: PL in vacuum. Dashed red curves: PL after $20 \mathrm{~min}$ in air, under constant laser excitation. Dash-dotted blue curve shows the difference between the black and the red curves and the dotted green line shows the intensity ratio between them (air/ vacuum). In (a), logarithmic scale was used and a baseline was subtracted, as shown in inset, in order to extract the weak InGaN PL from the strong GaN PL. (For interpretation of the references to color in this figure legend, the reader is referred to the web version of this article.) 
To confirm the role played by oxygen, we submitted the samples, under constant laser excitation, to cycles of (i) exposure to industrial grade purity $\mathrm{N}_{2}\left(\mathrm{O}_{2}<0.01 \mathrm{ppm} ; \mathrm{H}_{2} \mathrm{O}<0.02 \mathrm{ppm} ; \mathrm{CO} /\right.$ $\mathrm{CO}_{2}<0.5 \mathrm{ppm}$ ) for at least $20 \mathrm{~min}$, followed by (ii) exposure to vacuum for similar times. None of the cycles produced PL intensity changes larger than 5\%, contrary to the drastic changes provoked by air. Therefore, oxygen is clearly involved in the quenching mechanism, of which a modeling was proposed in Refs. $[1,20]$. From the viewpoint of band structure effects, this model has similarities with the one proposed by Calarco et al. [28] to explain transport measurements in GaN NWs. The model suggests that the observed PL quenching results from the decrease of the volume in which stable excitons can exist, because they are ionized by a strong near-surface electric field. The critical ionization field for excitons in GaN can be roughly estimated as $F_{\mathrm{C}}=E_{\mathrm{B}} /\left(e \cdot a_{\mathrm{B}}\right) \sim 10^{5} \mathrm{~V} / \mathrm{cm}$, where $E_{\mathrm{B}}$ and $a_{\mathrm{B}}$ are the exciton binding energy $(\sim 26 \mathrm{meV})$ and Bohr radius $(\sim 3 \mathrm{~nm})$. Following this model, our experiments indicate that, after exposure to air, the volume of the surface "exciton ionization" layer would correspond to $\sim 90 \%$ of the total NC volume. The observation by Pfüller et al. [27] of an increase of the recombination rate of donor-bound excitons at low temperatures, induced by oxygen desorption, encourages us to favor such a model based on the presence of surface electric fields. Nevertheless, we cannot discard completely the possibility that oxygen adsorption creates nonradiative recombination channels near the surface of the GaN NCs.

Let us now discuss the RT PL results obtained in InGaN-on-GaN NC samples, shown in Fig. 2. For small In content, the PL intensity of InGaN NCs was much weaker than the one from the underlying GaN NCs. For this reason, subtraction of a baseline was necessary to analyze the PL of InGaN NCs with In content of $\sim 10 \%$ (insert in Fig. 2(a)). For higher In contents (Fig. 2(b) and (c)), the InGaN related PL spectra are more clearly separated from the GaN one. Upon exposure to air, the PL from InGaN NCs showed much less quenching than in the case of GaN. In fact, the higher energy parts of the InGaN PL spectra were nearly unaffected by this exposure to air. On the other hand, our experiments reveal the existence of a lowerenergy contribution that is quenched by $\sim 80-90 \%$ : the spectrum of InGaN NCs does change. The insert in Fig. 2(a) shows a weak PL contribution at $2.68 \mathrm{eV}$, for the sample placed in vacuum: after exposure to air, this contribution loses intensity relatively to the higher energy side of the InGaN PL, at $2.83 \mathrm{eV}$. For the samples with higher In contents, it is easier to visualize the low-energy contribution: the difference between PL spectra in air and in vacuum is shown by the dash-dotted lines in Fig. 2(b) and (c). Clearly, this difference exhibits a peak situated typically $0.15 \mathrm{eV}$ below the PL peak in the vacuum.

\section{Discussion}

We need to discuss the differences between GaN and InGaN NCs along two lines: (1) the overall reduced quenching upon exposure to air for InGaN NCs and (2) the observation of a low-energy contribution in InGaN NC PL that does exhibit significant quenching.

(1) We can tentatively attribute the first observation to the localization of carriers at deep potential fluctuations, which exists in the ternary alloys but not in the binary compound [19]. The linewidths of the present PL spectra (several hundred $\mathrm{meV}$ ) provide an estimation of the overall range of those potential fluctuations. A localized electron-hole pair in a potential well of typically several tens of meV of depth, in InGaN, will be less easily split apart by an electric field of a few hundred $\mathrm{kV} / \mathrm{cm}$ than the free electron-hole pair in GaN, only bound by Coulomb interaction. Therefore, if a strong surface electric field is established upon oxygen accumulation, localized excitons are more robust than free ones against ionization. They are more robust, too, against capture by nonradiative recombination centers, if this turns out to be the mechanism at work upon oxygen adsorption. Carrier localization can be also invoked as a limiting factor of the oxygen adsorption itself, since the transfer of charged carriers towards the surface was proposed as a crucial mechanism favoring this adsorption. If the carrier mobility towards the surface is hindered by potential fluctuations, the photochemical process of adsorption can be strongly reduced. Finally, we must mention that InGaN is well known to have a stronger residual n-doping than GaN. This may drastically reduce the thickness of the surface space-charge region [28] and therefore minimize the overall sensitivity of the PL in InGaN NCs to oxygen adsorption.

(2) As for the existence of a lower-energy PL contribution that quenches under oxygen exposure, we have to make a preliminary remark: from the above explanation, we can infer that the deeper an exciton is localized, the less it will be affected by surface field effects. Therefore, one would expect 
the lower-energy part of the PL spectrum to be less sensitive to surface effects, contrary to what we show in Fig. 2. To explain this apparent contradiction, several mechanisms can be invoked. The first explanation supposes the existence, in each of the NCs, of a core and a surface region, the latter being responsible for the lower-energy PL contribution. The simplest reason for this could be In segregation or any type of growth mechanism that would result in In-rich lateral surfaces with PL at lower energy. We do not discard this possibility, though recently reported results rather indicate the presence of a GaN shell around similar InGaN NCs [29]. Another way to obtain lower-energy PL close to the surface is to consider a constant average In content but a gradient of strain, for example via strain relaxation mechanisms during growth. The core of the InGaN NC would be compressively strained by latticematching on the underlying GaN NC (which is unstrained, as proved by the excitonic transition energies). This compression would result in higher PL energy, for the core region, whereas strain relaxation near the surface would result in lower PL energies.

The second explanation is rather related to different average In compositions in different NCs, as seems to be indicated by preliminary cathodoluminescence results. Though not yet fully established, the dynamics of the growth mechanisms could lead, for example, to a dependence of In incorporation on the diameter of the underlying GaN NC. If the PL sensitivity to air exposure is also diameter-dependent, then our results could also arise from that dependence. Why the higher In composition would then correspond to higher sensitivity to oxygen adsorption, still has to be understood, but this possibility should not be neglected at this stage.

Further structural characterization is needed to ascertain any of these hypotheses: insight into local In content and strain, e.g. by high-resolution transmission electron microscopy, may provide the required information.

\section{Conclusions}

In summary, we have observed the influence of the atmospheric environment on the RT PL intensities of GaN and InGaN nanocolumns. We have shown that photoadsorption and photodesorption of oxygen induce strong changes in PL intensities. However, while the PL from GaN NCs placed in air ambient is quenched by $90 \%$ from its value in vacuum, the PL from InGaN NCs exhibits two clearcut contributions, of which only the lower-energy one is quenched. The overall PL intensity of InGaN NCs is, however, much less affected by oxygen adsorption than for GaN NCs. We tentatively assign this observation to carrier localization in the ternary alloy. Concerning the observation of two contributions in the PL spectrum of InGaN NCs, we have discussed the results in terms of composition or strain variation within each single NC and amongst the different NCs.

These results might be of importance in terms of applications to light-emitting devices, since we show that surface adsorption of oxygen affects more RT radiative recombination in GaN NCs than in InGaN NCs.

\section{Acknowledgments}

The authors wish to acknowledge partial financial support by the EU under FP7 contract SMASH CP-IP 228999-2, and by National Funding Agencies under contracts CAM/ P2009/ESP-1503, MICINN/ CSD2006-19 and MICINN/ PLE2009-0023.

\section{References}

[1] M.A. Reshchikov, A. Behrends, A. Bakin, A. Waag, J. Vac. Sci. Technol. B 27 (2009) 1688. and references therein.

[2] T.M. Babinec, B.J.M. Hausmann, M. Khan, Y. Zhang, J.R. Maze, P.R. Hemmer, M. Lončar, Nat. Nanotechnol. 5 (2010) 195.

[3] C.M. Lieber, Z.L. Wang, MRS Bull. 32 (2007) 99.

[4] R. Songmuang, O. Landré, B. Daudin, Appl. Phys. Lett. 91 (2007) 251902.

[5] A. Trampert, J. Ristić, U. Jahn, E. Calleja, K.H. Ploog, Inst. Phys. Conf. Ser. 180 (2003) 167.

[6] M. Yoshizawa, A. Kikuchi, M. Mori, N. Fujita, K. Kishino, Jpn. J. Appl. Phys. 36 (1997) L459.

[7] M.A. Sánchez-García, E. Calleja, E. Monroy, F.J. Sánchez, F. Calle, E. Muñoz, R. Beresford, J. Cryst. Growth 183 (1998) 23.

[8] E. Calleja, M.A. Sánchez-García, F.J. Sánchez, F. Calle, F.B. Naranjo, E. Muñoz, U. Jahn, K.H. Ploog, Phys. Rev. B 62 (2000) 16826. 
[9] L. Cerrutti, J. Ristić, S. Fernández-Garrido, E. Calleja, A. Trampert, K.H. Ploog, S. Lazić, J.M. Calleja, Appl. Phys. Lett. 88 (2006) 213114.

[10] N. Wang, Y. Cai, R.Q. Zhang, Mater. Sci. Eng. R 60 (2008) 1.

[11] S. Fernăndez-Garrido, J. Grandal, E. Calleja, M.A. Sánchez-Garcia, D. López-Romero, J. Appl. Phys. 106 (2009) 126102.

[12] H. Sekiguchi, K. Kishino, A. Kikuchi, Appl. Phys. Lett. 96 (2009) 231104.

[13] A.P. Vajpeyi, A.O. Ajagunna, K. Tsagaraki, M. Androulidaki, A. Georgakilas, Nanotechnology 20 (2009) 325605

[14] C.-C. Hong, H. Ahn, C.-Y. Wu, S. Gwo, Opt. Express 17 (2009) 17227.

[15] Y.-L. Chang, J.L. Wang, F. Li, Z. Mi, Appl. Phys. Lett. 96 (2010) 013106.

[16] H.-M. Kim, W.C. Lee, T.W. Kang, K.S. Chung, C.S. Yoon, C.K. Kim, Chem. Phys. Lett. 380 (2003) 181.

[17] Y. Sun, Y.-H. Cho, H.-M. Kim, T.W. Kang, Appl. Phys. Lett. 87 (2005) 093115

[18] T. Kuykendall, P. Ulrich, S. Aloni, P. Yang, Nat. Mater. 6 (2007) 951.

[19] S.F. Chichibu, A. Uedono, T. Onuma, B.A. Haskell, A. Chakraborty, T. Koyama, P.T. Fini, S. Keller, S.P. Denbaars, J.S. Speck, U.K. Mishra, S. Nakamura, S. Yamaguchi, S. Kamiyama, H. Amano, I. Akasaki, J. Han, T. Sota, Nat. Mater. 5 (2006) 810.

[20] M. Foussekis, A.A. Baski, M.A. Reshchikov, Appl. Phys. Lett. 94 (2009) 162116.

[21] Y.Z. Zhang, H.P. He, Y.Z. Jin, B.H. Zhao, Z.Z. Ye, H.P. Tang, J. Appl. Phys. 104 (2008) 103529.

[22] L. Wischmeier, T. Voss, I. Rückmann, J. Gutowski, A.C. Mofor, A. Bakin, A. Waag, Phys. Rev. B 74 (2006) 195333.

[23] O. Brandt, C. Pfüller, C. Chèze, L. Geelhaar, H. Riechert, Phys. Rev. B 81 (2010) 045302.

[24] P. Corfdir, P. Lefebvre, J. Ristić, P. Valvin, E. Calleja, J.D. Ganière, B. Deveaud, J. Appl. Phys. 105 (2009) 013113.

[25] For a review, see, e.g., J.S. Wright, W. Lim, D.P. Norton, S.J. Pearton, F. Ren, J.L. Johmson, A. Ural, Semicond. Sci. Technol. 25 (2010) 024002 and references therein.

[26] U. Jahn, private communication.

[27] C. Pfüller, O. Brandt, F. Grosse, T. Flissikowski, C. Chèze, V. Consonni, L. Geelhaar, H.T. Grahn, H. Riechert, Phys. Rev. B 82 (2010) 045320

[28] R. Calarco, M. Marso, T. Richter, A.I. Aykanat, R. Meijers, Av.d. Hart, T. Stoica, H. Lüth, Nano Lett. 5 (2005) 981.

[29] C. Bougerol, A. Grenier, G. Tourbot, D.S. Giao, B. Gayral, D. Cooper, B. Daudin, Communication at the International Workshop on Nitride Semiconductors (IWN 2010), Tampa, USA, sept. 2010. To be published in Phys. Status Solidi C. 